\title{
Using Channel State Feedback to Achieve Resilience to Deep Fades in Wireless Networked Control Systems
}

\author{
Bin $\mathrm{Hu}$ and M.D. Lemmon
}

\begin{abstract}
Wireless networked control systems (WNCS) consist of several dynamical systems that exchange information over a wireless radio (RF) communication network. These RF networks are subject to deep fades where the effective link throughput drops precipitously. Deep fading negatively impacts WNCS performance and stability, but in many applications the probability of a deep fade is a function of the system state. This suggests that one can use channel state information (CSI) as a feedback signal to recover some of the performance lost. This paper derives necessary and sufficient conditions for the almost sure stability of WNCS in the presence of deep fading. These conditions relate the channel's state to the WNCS's convergence rate. This paper uses this fact to reconfigure WNCS controllers to recover system performance in the presence of such fades. The results are illustrated using a leader-follower scenario found in vehicle-to-vehicle ( $\mathrm{V} 2 \mathrm{~V})$ applications.
\end{abstract}

\section{INTRODUCTION}

Wireless networked control systems (WNCS) consist of several dynamical systems that coordinate their behavior by exchanging information over a wireless radio (RF) communication network. Examples of such systems are found in smart transportation [1] applications that anticipate automobiles exchanging information to coordinate their maneuvers. These RF communication channels, however, are subject to deep fades where the channel's throughput drops precipitously and remains low for an extended interval of time. Such fades clearly limit the ability of WNCS subsystems to successfully coordinate their actions. This lack of coordination has obvious negative impacts on the safety of smart transportation applications which could be addressed by developing WNCS that are resilient [2] to deep fading in the sense that the system detects the fade and then adapts its controller to maintain some minimum performance level.

Channel fading is characterized in terms of the channel gain; the ratio of the received signal strength over the transmitted signal strength. This gain is often modeled as an independent and identically distributed (i.i.d.) random process having either a Rayleigh or Rician distribution [3]. This channel model is inadequate for two reasons. In the first place, fading possesses a memory effect which suggests it is better modeled as a Markov process [4] with two states for a high gain condition and a low gain condition. The transition to the low gain state represents a deep fade. In the second place, this model ignores the potential dependence of the channel state on the states of the WNCS subsystems. Vehicleto-vehicle (V2V) applications provide an example in which the channel state is a function of the velocity and position of both vehicles [5], [6], [7], [8].

The loss of information resulting from a deep fade will negatively impact the performance achievable by WNCS subsystems. Prior work [9], [10], [11], [12], [13] characterized the minimum stabilizing bit rate for linear timeinvariant (LTI) systems assuming constant channel gain. But as noted above, the assumption of a constant channel gain is overly simplistic since it ignores channel fading. Initial attempts to study the impact of time-varying channels on a control system's mean square stability have recently appeared [14], [15], but this work has assumed the channel gain is decoupled from the dynamics of the control system. This paper examines a more realistic fading model in which the channel is exponentially bursty [16], [17] and is dependent on the physical plant's state. In particular, this paper characterizes conditions that the physical state of the plant and the exponential bursty channel have to satisfy to assure the stronger stability notion of almost sure stability. The paper uses this characterization to propose adaptive schemes that switch feedback controllers in response to changes in the communication channel's state. The results are illustrated on a leader-follower system motivated by smart transportation applications.

\section{MATHEMATICAL PRELIMINARIES}

Let $\mathbb{R}$ and $\mathbb{Z}$ denote the set of real numbers and integers, respectively. The sets of positive reals and integers are denoted as $\mathbb{R}^{+}$and $\mathbb{Z}^{+}$, respectively. Euclidean $n$-space is denoted as $\mathbb{R}^{n}$. The $\infty$-norm on the vector $x \in \mathbb{R}^{n}$ is $|x|=$ $\max \left|x_{i}\right|: 1 \leq i \leq n$, and the corresponding induced matrix norm $\|A\|=\max _{1 \leq i \leq n} \sum_{j=1}^{n}\left|A_{i}^{j}\right|$. Given a vector $x \in \mathbb{R}^{n}$, we let $x_{i} \in \mathbb{R}$ for $i=1,2, \ldots, n$ denote the $i$ th element of vector $x$. We let $f(\cdot): \mathbb{R} \rightarrow \mathbb{R}^{n}$ denote a function mapping the real line onto vectors in $\mathbb{R}^{n}$. We let $f(t) \in \mathbb{R}^{n}$ denote the value that function $f$ takes at time $t \in \mathbb{R}$. The left-hand limit at $t \in \mathbb{R}$ of a function $f(\cdot): \mathbb{R} \rightarrow \mathbb{R}^{n}$ is denoted as $f\left(t^{-}\right)$.

Consider a dynamical system whose state trajectory $x(\cdot)$ : $\mathbb{R} \rightarrow \mathbb{R}^{n}$ satisfies the following differential equation

$$
\dot{x}(t)=A x(t)+B u(t), \quad x(0)=x_{0}
$$

for all $t \in \mathbb{R}^{+}$where $u(\cdot): \mathbb{R} \rightarrow \mathbb{R}^{m}$ is an input signal, $x_{0} \in \mathbb{R}^{n}$ is the initial state, and the matrices $A$ and $B$ are appropriately dimensioned. Let the output of this system be the signal $y(\cdot): \mathbb{R} \rightarrow \mathbb{R}^{q}$ that satisfies

$$
y(t)=C x(t)+w(t)
$$

where $C$ is an appropriately dimensioned matrix, $w(\cdot): \mathbb{R} \rightarrow$ $\mathbb{R}^{q}$ is the stochastic disturbance process. For any $T>0$, let $\Phi(T)$ denote the state transition matrix, and its $\infty$-induced norm is denoted as $\|\Phi(T)\|$. 
The probability of a random variable is denoted by $\operatorname{Pr}(\cdot)$ and its expectation by $\mathbb{E}(\cdot)$. Consider a random process $\left\{x_{t}\right\}$. We say this process is almost sure convergent to 0 if for all $\varepsilon>0$ there exists $\delta>0$ and $T>0$ such that

$$
\operatorname{Pr}\left\{\operatorname{lim\operatorname {sup}_{t>T}}\left|x_{t}\right|>\varepsilon\right\}<\delta
$$

One often writes this as $\operatorname{Pr}\left\{\left|x_{t}\right| \rightarrow 0\right\}=1$. If the random process $\left\{x_{t}\right\}$ is generated by the system equations (1-2) then we say this system is almost sure stable.

The system in equations (1-2) is almost sure asymptotically observable if for any $T>0$ one can use the system inputs and outputs to generate a sequence $\left\{\hat{x}_{k}, L_{k}\right\}_{k=1}^{\infty}$ such that such that $e_{k}=\left|x(k T)-\hat{x}_{k}\right|<L_{k}$ for all $k=1,2, \ldots, \infty$ and $L_{k} \rightarrow 0$ as $k \rightarrow \infty$.

The concept of almost sure asymptotic observability may be viewed as a probabilistic extension of the asymptotic observability concept found in [12]. Similar to what is found in [12], a system's almost sure asymptotic observability can be shown to be sufficient for the system to be almost sure stable. This paper establishes that result and then uses it to determine conditions for the almost sure stability of the system under study.

\section{SYSTEM MODEL}

The proposed system under study is shown in Figure 1. This figure shows a plant whose state trajectory $x(\cdot): \mathbb{R} \rightarrow \mathbb{R}^{n}$ satisfies the system equations (1). The input signal $u$ is generated by a controller and its output is the state vector. The plant's state is sampled by an encoder at a sequence of time instants $\left\{\tau_{k}\right\}_{k=1}^{\infty}$. The encoder maps the system state $x\left(\tau_{k}\right)$ onto a codeword, $c(k)=\left\{b_{i}(k)\right\}_{i=1}^{\bar{R}}$ which is a collection of $\bar{R} \in \mathbb{Z}^{+}$blocks of bits with block length $n$. In other words $b_{i}(k) \in\{0,1\}^{n}$ is the $i$ th block in the codeword generated by the encoder at sampling time $\tau_{k}$. The codeword is transmitted over the channel. The output of the channel is a codeword, $\hat{c}(k)=\left\{\hat{b}_{i}(k)\right\}_{i=1}^{R_{k}}$ that is received by the decoder at time $a_{k}=\tau_{k}+\Delta$. The interval $\Delta>0$ represents a finite time delay which is constant and known to both the encoder and decoder. The received codeword consists of $R_{k} \leq \bar{R}$ blocks and we assume that the $i$ th received block, $\hat{b}_{i}(k)$, equals the $i$ th transmitted block $b_{i}(k)$ for $i=1,2, \ldots, R_{k}$. In other words, the first $R_{k}$ blocks of bits in the transmitted codeword are reliably received by the decoder. The decoder then uses the received bits in $\hat{c}(k)$ to construct an estimate for the plant's state. We denote this estimate as $\hat{x}_{k}(k=1,2, \ldots, \infty)$ and the controller uses this state to generate the control signal $u(\cdot): \mathbb{R} \rightarrow \mathbb{R}^{m}$ used by the plant. More detailed descriptions of the channel, encoder/decoder, and controller blocks are given below.

Channel Model: The stochastic nature of the channel is captured by the number of received bits, $n R_{k}$, at time instant $a_{k}$. In particular, we assume that $R_{k}$ is a random process and we let $r_{k}=\frac{n R_{k}}{\Delta}$ where $\Delta=a_{k}-\tau_{k}$ denote the instantaneous bit rate for the $k$ th transmission. Let $h_{i}(\cdot): \mathbb{R}^{+} \rightarrow \mathbb{R}^{+}(i=$ $1,2)$ and $\zeta_{2}(\cdot): \mathbb{R}^{+} \rightarrow \mathbb{R}^{+}$be continuous, non-negative and monotone decreasing functions. Let $\zeta_{1}(\cdot): \mathbb{R}^{+} \rightarrow \mathbb{R}^{+}$be

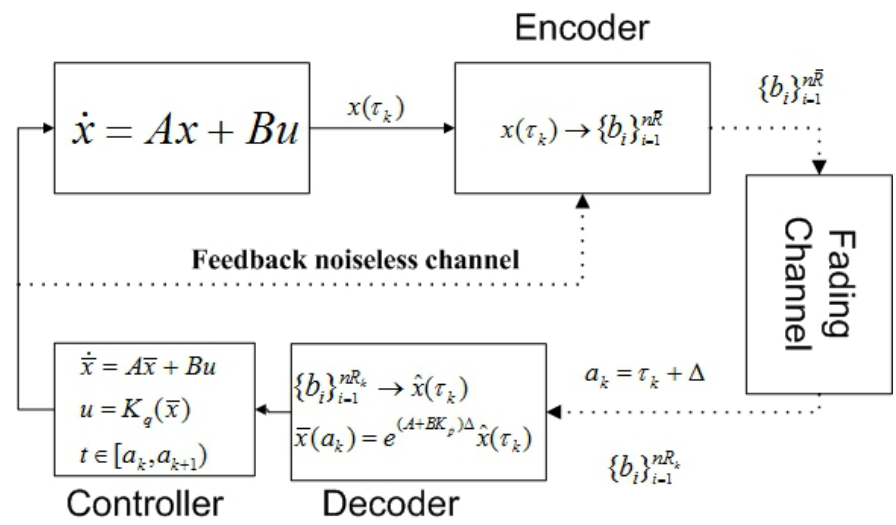

Fig. 1. System Structure

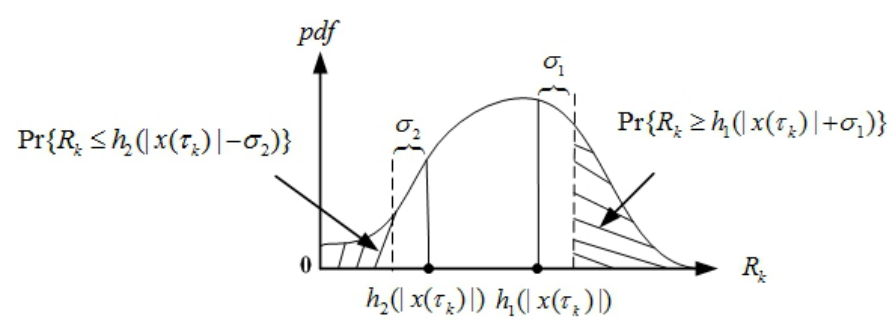

Fig. 2. Probability bounds for the channel model

continuous,non-negative and monotone increasing function. We assume that the tail of $R_{k}$ 's probability distribution satisfies the following exponential bounds

$$
\begin{aligned}
& \operatorname{Pr}\left\{R_{k} \geq h_{1}\left(\left|x\left(\tau_{k}\right)\right|\right)+\sigma_{1}\right\} \leq e^{-\zeta_{1}\left(\left|x\left(\tau_{k}\right)\right|\right) \sigma_{1}} \\
& \operatorname{Pr}\left\{R_{k} \leq h_{2}\left(\left|x\left(\tau_{k}\right)\right|\right)-\sigma_{2}\right\} \leq e^{-\zeta_{2}\left(\left|x\left(\tau_{k}\right)\right|\right) \sigma_{2}}
\end{aligned}
$$

for all real $\sigma \geq 0$ and $\sigma_{2} \in\left[0, h_{2}\left(\left|x\left(\tau_{k}\right)\right|\right)\right]$.

The tail of $R_{k}$ 's probability distribution is shown in figure 2. The left tail of the distribution characterizes the instantaneous low bit rate region while the right tail represents the instantaneous high bit rate region. The low bit rate region at $k$ th transmission interval is the set of all $R_{k}$ less than $h_{1}\left(\left|x\left(\tau_{k}\right)\right|\right)$. One may view $h_{1}\left(\left|x\left(\tau_{k}\right)\right|\right)$ as a threshold for the low bit rate region, and varies as a function of the system state. The high bit rate region is constructed in a similar manner.

The probability bounds in (3-4) may be viewed as slight modifications of the exponentially bounded burstiness(EBB) model [16]. The only difference lies in our characterization of system state's impact on the model. It is well established that the two state Markov chain commonly used to characterize the fading channel, can be approximated by the exponentially bounded burstiness (EBB) model [16]. Although the EBB model may turn out be conservative because it bounds the occurrence of rare events, it is convenient to use for establishing the system's almost sure stability

The monotone decreasing functions $h_{i}(\cdot), i=1,2$ are introduced to model the basic relationship between channel state and control system's state. This is motivated by the fact that increasing the system state's norm will decrease the channel state and vice versa. $\zeta_{i}(\cdot), i=(1,2)$ is used to characterize 
the exponent in the probability bounds. The further the state is from the equilibrium, the less likely that the high data rate region will expand, but more likely the low data rate will move toward the origin. It models the fact that channel is more prone to exhibit deep fading when the system states are further from the equilibrium.

Controller: At time instant $a_{k}$, the controller receives an estimate of the sampled system state at time instant $\tau_{k}$. This estimate is denoted as $\hat{x}_{k}$. To generate the control over the time interval $\left[a_{k}, a_{k+1}\right]$, the controller first selects a control gain, $K \in \mathscr{K}$ where $\mathscr{K}=\left\{K_{1}, K_{2}, \cdots, K_{N}\right\}$ is a collection of state feedback gains. We let $K(k)$ denote the controller that was selected over the $k$ th action interval. The controller then generates an estimated state trajectory, $\bar{x}(\cdot):\left[a_{k}, a_{k+1}\right] \rightarrow \mathbb{R}^{n}$ that satisfies the ODE,

$$
\dot{\bar{x}}(t)=(A+B K(k)) \bar{x}(t), \quad \bar{x}\left(a_{k}\right)=e^{(A+B K(k-1)) \Delta_{\hat{x}}}
$$

and the control is then

$$
u(t)=K(k) \bar{x}(t)
$$

for $t \in\left[a_{k}, a_{k+1}\right]$. These equations apply for all $k=1,2, \ldots, \infty$.

Encoder/Decoder: The encoder/decoder is characterized by a sequence of $\left\{\left(\hat{x}_{k}, L_{k}\right)\right\}_{k=1}^{\infty}$ which represent the estimated state, $\hat{x}_{k}$, and the size of the uncertainty set $L_{k}$ associated with the $k$ th consecutive transmission. It is assumed that at $k=0$, both encoder and decoder have the same initial state name $\hat{x}_{0}=0$ and $L_{0}=\bar{L}$. The encoder then maps the current sampled state, $x\left(\tau_{k}\right)$, onto the bits $b_{i}(k)(i=1,2, \ldots, n \bar{R})$ according to the following series expansion of the sampled state

$$
\begin{aligned}
& x\left(\tau_{k}\right)=\bar{x}\left(\tau_{k}\right)+\|\Phi(T)\| L_{k-1} \sum_{j=1}^{\infty} \frac{1}{2^{j}} \bar{b}_{j} \\
& \bar{x}\left(\tau_{k}\right)=e^{(A+B K(k-1))(T-\Delta)} \cdot e^{(A+B K(k-2)){ }_{x}} \hat{x}_{k-1}
\end{aligned}
$$

where $\|\Phi(T)\|=\left\|e^{A T}\right\|$ and $\bar{b}$ is an $n$ vector whose components are \pm 1 . This vector is encoded with $n$ bits with bit 0 representing value -1 and bit 1 representing value 1 , and the transmitted code word consists of the first $n \bar{R}$ bits.

We assume that the the decoder receives the first $n R_{k}$ bits (or rather the first $R_{k}$ blocks) in the transmitted codeword and the reconstructed state is then

$$
\hat{x}_{k}=\bar{x}\left(\tau_{k}\right)+\|\Phi(T)\| L_{k-1} \sum_{j=1}^{R_{k}} \frac{1}{2^{j}} \bar{b}_{j}
$$

Figure 3 shows a two-dimensional system with $\bar{R}=2$ blocks ( 4 bits) transmitted through the channel, and only one block $R_{k}=1$ ( 2 bits) is received. By using the encoder scheme in equation (5), a codeword $c(k)=\{[1,1],[1,1]\}$ is constructed and used to label the small square on the rightmost corner with true state inside. The decoder only receives the first block of the original codeword, i.e. $\hat{c}(k)=\{[1,1]\}$. The reconstruction equation in (6) gives the estimated state $\hat{x}_{k}$ marked as red dot in the larger square. It is clear from the picture that the reception of partial information can still guarantee the recovery of the sensor measurement but with

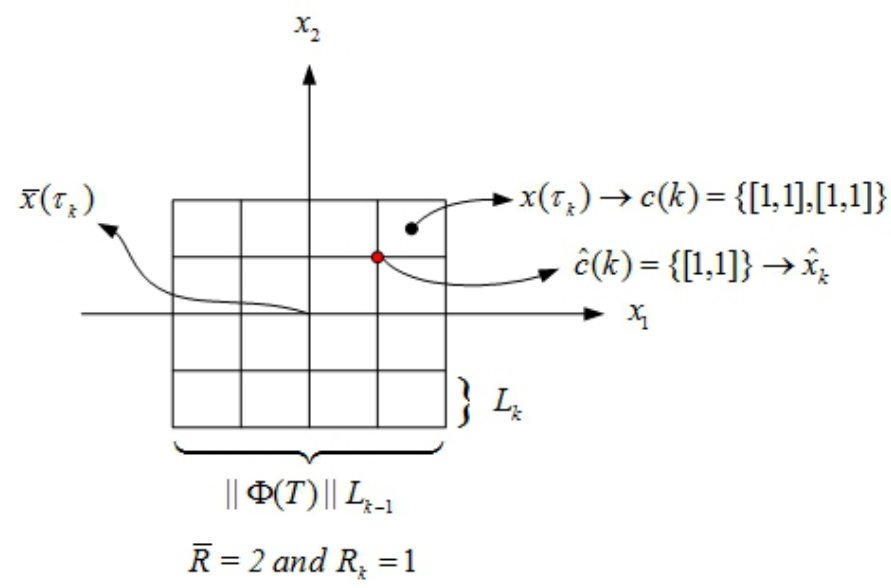

Fig. 3. Encoder and Decoder mapping

a reduced precision. Such reduced precision can be quantitatively bounded if the synchronization of the information is assured on both encoder and decoder.

From the encoding scheme in equation (5), the information needed to be synchronized includes the prior control gains $K(k-1)$ and $K(k-2)$ as well as the estimated state $\hat{x}_{k-1}$. As shown in figure 1, this information is obtained from decoder and controller through a feedback noiseless channel at each time interval $\left[\tau_{k}, a_{k}\right]$. In particular, encoder will construct the estimated state $\hat{x}_{k-1}$ by assessing the actual number of bits $n R_{k-1}$ received at $k-1$ th interval. The synchronization on $R_{k-1}$ can be achieved by using acknowledgement signal indicating the receipt of each block at the decoder side. As for $K(k-1)$ and $K(k-2)$, their corresponding indices will be sent to encoder provided encoder and decoder share the selection set for the controller.

Such synchronization ensures the encoder and decoder shares the estimation error bound at each time instant $\tau_{k}$ as follows,

$$
\begin{gathered}
\left|x\left(\tau_{k}\right)-\hat{x}_{k}\right| \leq 2^{-R_{k}}\|\Phi(T)\| L_{k-1} \\
\text { IV. MAIN RESULTS }
\end{gathered}
$$

\section{MAIN RESUlTS}

This section derives necessary and sufficient characterization for the almost sure convergence of sequence $\left\{L_{k}\right\}_{k=0}^{\infty}$, and then proves that the convergence of this sequence guarantees the almost sure asymptotic observability for the system defined in equation (1). Under a dwell time assumption (which represents the minimum time between controller switches), we further show that the almost sure asymptotic observability implies almost sure asymptotic stability for the control system in equation (1).

The following proposition bounds the quantization error generated by the proposed encoder/decoder scheme. The proof follows that found in [12].

Proposition 4.1: Given the sequences $\left\{\tau_{k}\right\}_{k=0}^{\infty}$ and $\left\{a_{k}\right\}_{k=0}^{\infty}$, encoder and decoder scheme in equations (5-6), suppose the initial state $x(0) \in[-\bar{L}, \bar{L}]^{n}, L_{0}>0$, if the sequence $\left\{L_{k}\right\}_{k=0}^{\infty}$ is constructed by the following equation

$$
L_{k+1}=2^{-R_{k+1}}\|\Phi(T)\| L_{k}
$$


then the quantization error can be bounded as,

$$
\left\|x\left(\tau_{k}\right)-\hat{x}\left(\tau_{k}\right)\right\| \leq L_{k}, \forall k \geq 0
$$

with initial value $L_{0}=2^{-R_{0}} \bar{L}$, where $R_{k}$ is the number of blocks received during time interval $\left[\tau_{k}, a_{k}\right)$.

\section{A. Almost sure asymptotic observability}

The following lemma establishes a necessary condition for the almost sure asymptotic convergence of sequence $\left\{L_{k}\right\}_{k=0}^{\infty}$ with convergence rate $\delta \in(0,1]$. The necessary condition is derived based on the probability bound on the high data rate region. The necessity comes from the fact that if the system performance cannot be assured under the high data rate scenario, there is no way for the system performance to be better under other scenarios.

Lemma 4.2: Consider the system in equation (1), suppose right tail of $R_{k}$ probability distribution satisfies the probability bound in (3), then a necessary condition to ensure

$$
\mathbb{E}\left(L_{k} \mid L_{k-1}\right) \leq \delta L_{k-1}, \forall k \in \mathbb{Z}^{+}
$$

is

$$
\left|x\left(\tau_{k}\right)\right| \leq G_{1}^{-1}\left(\frac{\delta}{\|\Phi(T)\|}\right), \forall k \in \mathbb{Z}
$$

where $\|\Phi(T)\|=\left\|e^{A T}\right\|, \delta \in(0,1]$ and function $G_{1}(y)=$ $2^{-h_{1}(y)} \cdot \frac{\zeta_{1}(y)}{\zeta_{1}(y)+\ln 2}$.
Proof: $\operatorname{In}$

Proof: In order to prove the necessary condition ensuring $\mathbb{E}\left(L_{k} \mid L_{k-1}\right) \leq \delta L_{k-1}$, we prove the case that the $\mathbb{E}\left(L_{k} \mid L_{k-1}\right)>\delta L_{k-1}$ holds when condition (9) is violated, i.e.

$$
\left|x\left(\tau_{k}\right)\right|>G_{1}^{-1}\left(\frac{\delta}{\|\Phi(T)\|}\right), \forall k \in \mathbb{Z}
$$

First, Let us consider the condition that makes $\mathbb{E}\left(L_{k} \mid L_{k-1}\right)>$ $\delta L_{k-1}$ hold, i.e.

$$
\|\Phi(T)\| E\left[2^{-R_{k}}\right]>\delta
$$

Let $\tilde{R}:=2^{-R_{k}}, h_{1 k}:=h_{1}\left(\left|x\left(\tau_{k}\right)\right|\right)$ and $\zeta_{1 k}:=\zeta_{1}\left(\left|x\left(\tau_{k}\right)\right|\right)$. The probability bound in (3) implies

$$
\operatorname{Pr}\left\{2^{-R_{k}} \leq 2^{-\left(h_{1 k}+\sigma_{1}\right)}\right\}=\operatorname{Pr}\left\{R_{k} \geq h_{1 k}+\sigma_{1}\right\} \leq e^{-\zeta_{1 k} \sigma_{1}}
$$

Now, the expectation of $2^{-R_{k}}$ can be written as

$$
\begin{aligned}
\mathbb{E}(\tilde{R}) & =\int_{0}^{1} \operatorname{Pr}\{\tilde{R}>x\} d x \\
& =1-\int_{0}^{1} \operatorname{Pr}\{\tilde{R} \leq x\} d x
\end{aligned}
$$

Since

$$
\begin{aligned}
\int_{0}^{1} \operatorname{Pr}\{\tilde{R} \leq x\} d x & =\int_{0}^{2^{-h_{1 k}}} \operatorname{Pr}\{\tilde{R} \leq x\} d x+\int_{2^{-h_{1 k}}}^{1} \operatorname{Pr}\{\tilde{R} \leq x\} d x \\
& =\int_{0}^{\infty} \operatorname{Pr}\left\{\tilde{R} \leq 2^{-h_{1 k}-\sigma_{1}}\right\}\left(2^{-h_{1 k}} \cdot 2^{-\sigma_{1}} \ln 2 \cdot d \sigma_{1}\right. \\
& +1-2^{-h_{1 k}} \\
& \leq \int_{0}^{\infty} e^{-\zeta_{1 k} \sigma_{1}} 2^{-h_{1 k}} 2^{-\sigma_{1}} \ln 2 \cdot d \sigma_{1} \\
& +1-2^{-h_{1 k}}
\end{aligned}
$$

we have the following lower bound of $\mathbb{E}(\tilde{R})$

$$
\begin{aligned}
\mathbb{E}(\tilde{R}) & \geq 2^{-h_{1 k}}-2^{-h_{1 k}} \ln 2 \int_{0}^{\infty} e^{-\zeta_{1 k} \sigma_{1}} 2^{-\sigma_{1}} d \sigma_{1} \\
& =2^{-h_{1 k}} \cdot \frac{\zeta_{1 k}}{\zeta_{1 k}+\ln 2} \\
& \triangleq G_{1}\left(\left|x\left(\tau_{k}\right)\right|\right)
\end{aligned}
$$

Taking the derivative of $G_{1}(y)=2^{-h_{1}(y)} \cdot \frac{\zeta_{1}(y)}{\zeta_{1}(y)+\ln 2}$ with respect to $y$, we obtain

$$
\begin{aligned}
\dot{G}_{1} & =-\ln 2 \cdot \dot{h}_{1} 2^{-h_{1}}\left[1-\frac{\ln 2}{\zeta_{1}+\ln 2}\right]+2^{-h_{1}} \frac{\ln 2 \cdot \dot{\zeta}_{1}}{\left(\zeta_{1}+\ln 2\right)^{2}} \\
& =\frac{\ln 2 \cdot 2^{-h_{1}}}{\zeta_{1}+\ln 2}\left[-\zeta_{1} \cdot \dot{h}_{1}+\frac{\dot{\zeta}_{1}}{\zeta_{1}+\ln 2}\right]
\end{aligned}
$$

Note that the function $h_{1}$ is positive and monotone decreasing while function $\zeta_{1}$ is positive monotone increasing. It is clear that $\dot{G}_{1}>0$ holds. This implies the violation of the condition in (9) will lead to $\mathbb{E}(\tilde{R})>\frac{\delta}{\|\Phi(T)\|}$, which implies $\mathbb{E}\left(L_{k} \mid L_{k-1}\right)>\delta L_{k-1}$. Therefore, condition (9) is a necessary condition for $\mathbb{E}\left(L_{k} \mid L_{k-1}\right) \leq \delta L_{k-1}$ to hold. The proof is complete.

The following lemma derives a sufficient condition for the controller so that the necessary condition derived in lemma 4.2 can be achieved.

Lemma 4.3: Consider the system in equation (1) with a family of control gain matrices $\left\{K_{i}\right\}_{i=1}^{N}$, suppose right tail of $R_{k}$ probability distribution satisfies the probability bound in (3), if the selected controller $K \in\left\{K_{i}\right\}_{i=1}^{N}$ satisfies the condition

$$
\left\|A_{K}\right\| \leq \frac{1}{T-\Delta} \ln \frac{G_{1}^{-1}\left(\frac{\delta}{\|\Phi(T)\|}\right)-\|\Phi(T)\| L_{k-1}}{\left|\hat{x}\left(a_{k-1}\right)\right|}
$$

the necessary condition derived in lemma 4.2, i.e. $\left|x\left(\tau_{k}\right)\right| \leq$ $G_{1}^{-1}\left(\frac{\delta}{\|\Phi(T)\|}\right), \forall k \in \mathbb{Z}$ is achieved.

Proof: Consider the upper bound of $\left|x\left(\tau_{k}\right)\right|$ that is derived from the dynamics of the quantization error. Using encoding and decoding scheme in (5-6), the quantization error at time $\tau_{k}^{-}$can be bounded as

$$
\left|x\left(\tau_{k}^{-}\right)-\hat{x}\left(\tau_{k}^{-}\right)\right| \leq\|\Phi(T)\| L_{k-1}
$$

Because of the continuity of original state at time $\tau_{k}^{-}, x\left(\tau_{k}\right)=$ $x\left(\tau_{k}^{-}\right)$, then

$$
\left|x\left(\tau_{k}\right)\right|=\left|x\left(\tau_{k}^{-}\right)\right| \leq\left|\hat{x}\left(\tau_{k}^{-}\right)\right|+\|\Phi(T)\| L_{k-1}
$$

Since controller reconfiguration will only be conducted at time sequence $\left\{a_{k}\right\}_{k=0}^{\infty}, \hat{x}\left(\tau_{k}^{-}\right)$can be calculated as follows if a controller $K$ is selected,

$$
\hat{x}\left(\tau_{k}^{-}\right)=e^{(A+B K)(T-\Delta)} \cdot \hat{x}\left(a_{k-1}\right)
$$

Now, let the controller $K$ satisfies the condition (13), consider the inequalities (16-15), we have $\left|x\left(\tau_{k}\right)\right| \leq G_{1}^{-1}\left(\frac{\delta}{\|\Phi(T)\|}\right), \forall k \in$ $\mathbb{Z}$ holds. The proof is complete.

It is worth noting that the necessary condition may turn out to be optimistic since it only captures the high data rate 
region. The following lemma establish sufficient conditions for the same performance specification. In contrast, the sufficient conditions are derived based on the probability bound on the low bit rate region, which is the worst case scenario.

Lemma 4.4: Consider the system in equation (1), and suppose the left tail of $R_{k}$ 's distribution satisfies the probability bound (4), then a sufficient condition to ensure

$$
\mathbb{E}\left(L_{k} \mid L_{k-1}\right) \leq \delta L_{k-1}, \forall k \in \mathbb{Z}^{+}
$$

is

$$
\left|x\left(\tau_{k}\right)\right| \leq G_{2}^{-1}\left(\frac{\delta}{\|\Phi(T)\|}\right), \forall k \in \mathbb{Z}
$$

where $\|\Phi(T)\|=\left\|e^{A T}\right\|, \delta \in(0,1]$ and function $G_{2}(y)=$ $e^{-h_{2}(y) \zeta_{2}(y)}\left(1+h_{2}(y) \zeta_{2}(y)\right)$.

Proof: Unlike the logic used to prove necessary condition, here, we want to a condition that ensures $\mathbb{E}\left(L_{k} \mid L_{k-1}\right) \leq$ $\delta L_{k-1}, \forall k \in \mathbb{Z}^{+}$. Establishing this is equivalent to proving

$$
\|\Phi(T)\| \mathbb{E}\left[2^{-R_{k}}\right] \leq \delta, \delta \in(0,1]
$$

Since we assume the probability bound (4) holds, we have

$$
\begin{array}{r}
\operatorname{Pr}\left\{2^{-R_{k}} \geq 2^{-h_{2}\left(\left|x\left(\tau_{k}\right)\right|\right)+\sigma_{2}}\right\} \leq e^{-\zeta_{2}\left(\left|x\left(\tau_{k}\right)\right|\right) \sigma_{2}} \\
\sigma_{2} \in\left[0, h_{2}\left(\left|x\left(\tau_{k}\right)\right|\right)\right]
\end{array}
$$

let $\tilde{R}_{k}:=2^{-R_{k}}, h_{2 k}:=h_{2}\left(\left|x\left(\tau_{k}\right)\right|\right)$ and $\zeta_{2 k}:=\zeta_{2}\left(\left|x\left(\tau_{k}\right)\right|\right)$, then

$$
\begin{aligned}
\mathbb{E}\left(\tilde{R}_{k}\right) & =\int_{0}^{1} \operatorname{Pr}\left\{\tilde{R}_{k}>x\right\} d x \\
& =\int_{0}^{2^{-h_{2 k}}} \operatorname{Pr}\left\{\tilde{R}_{k}>x\right\} d x \\
& +\int_{2^{-h_{2 k}}}^{1} \operatorname{Pr}\left\{\tilde{R}_{k}>x\right\} d x
\end{aligned}
$$

Following the same technique in lemma 4.2, we obtain

$$
\mathbb{E}\left(\tilde{R}_{k}\right) \leq 2^{-h_{2 k}}+2^{-h_{2 k}} \ln 2 \cdot \int_{0}^{h_{2 k}} e^{-\zeta_{2 k} \sigma_{2}} 2^{\sigma_{2}} \cdot d \sigma_{2}
$$

Let $C:=\int_{0}^{h_{2 k}} e^{-\zeta_{2 k} \sigma_{2}} 2^{\sigma_{2}} \cdot d \sigma_{2}$, integration by parts gives rise to

$$
C=\frac{1}{\ln 2}\left[\left.e^{-\zeta_{2 k} \sigma_{2}} 2^{\sigma_{2}}\right|_{0} ^{h_{2 k}}+\zeta_{2 k} C\right]
$$

Hence, we have

$$
C=\frac{e^{h_{2 k}\left(\ln 2-\zeta_{2 k}\right)}-1}{\ln 2-\zeta_{2 k}}>0
$$

Substituting equation (22) into inequality (20), $\mathbb{E}\left(\tilde{R}_{k}\right)$ is bounded by

$$
\mathbb{E}\left(\tilde{R}_{k}\right) \leq \frac{e^{-h_{2 k} \zeta_{2 k}} \ln 2-2^{-h_{2 k}} \zeta_{2 k}}{\ln 2-\zeta_{2 k}}
$$

Note that $2^{-h_{2 k}}=e^{-h_{2 k} \cdot \ln 2}$, the above upper bound can be further rewritten as

$$
\begin{aligned}
\mathbb{E}\left(\tilde{R}_{k}\right) & \leq \frac{e^{-h_{2 k} \zeta_{2 k}\left[\ln 2-e^{-h_{2 k} \cdot\left(\ln 2-\zeta_{2 k}\right)} \zeta_{2 k}\right]}}{\ln 2-\zeta_{2 k}} \\
& =e^{-h_{2 k} \zeta_{2 k}}\left[1+\zeta_{2 k} \cdot \frac{1-e^{-h_{2 k}\left(\ln 2-n \zeta_{2 k}\right)}}{\ln 2-\zeta_{2 k}}\right]
\end{aligned}
$$

It is easy to check that the right hand side of above inequality is bounded away from zero. Let $\varphi:=\ln 2-\zeta_{2 k}$, consider function $f(\varphi)=1-e^{-h_{2 k} \varphi}-h_{2 k} \varphi$, since

$$
\begin{aligned}
& \frac{d f(\varphi)}{d t}=h_{2 k}\left(e^{-h_{2 k} \varphi}-1\right) \leq 0, \varphi \geq 0 \\
& \frac{d f(\varphi)}{d t}=h_{2 k}\left(e^{-h_{2 k} \varphi}-1\right)>0, \varphi<0
\end{aligned}
$$

$f(\varphi)$ obtains maximum value at $\varphi=0$, i.e. $f(\varphi) \leq f(0)=0$. It implies

$$
\begin{aligned}
& 1-e^{-h_{2 k} \varphi} \leq h_{2 k} \varphi \\
\Rightarrow & \frac{1-e^{-h_{2 k} \varphi}}{\varphi} \leq h_{2 k}
\end{aligned}
$$

By applying above inequality, $\mathbb{E}\left(\tilde{R}_{k}\right)$ is further bounded as

$$
\mathbb{E}\left(\tilde{R}_{k}\right) \leq e^{-h_{2 k} \zeta_{2 k}}\left[1+h_{2 k} \zeta_{2 k}\right]
$$

Recall that $h_{2 k}=h_{2}\left(\left|x\left(\tau_{k}\right)\right|\right)$ and $\zeta_{2}\left(\left|x\left(\tau_{k}\right)\right|\right)$ are both nonnegative and monotone decreasing functions. Hence, function $g(y)=h_{2} \cdot \zeta_{2}$ is also nonnegative and monotone decreasing. Let $G_{2}(y)=e^{-h_{2}(y) \zeta_{2}(y)}\left(1+h_{2}(y) \zeta_{2}(y)\right)$,

$$
\frac{d G_{2}(y)}{d y}=-\frac{d g(y)}{d y} \cdot g(y) e^{-g(y)} \geq 0
$$

Hence, $G_{2}(y)$ is nonnegative and monotone increasing function. And we have

$$
\left.\mathbb{E}\left(\tilde{R}_{k}\right) \leq G_{2}\left(\| x\left(\tau_{k}\right)\right) \|\right)
$$

Now, it is clear that if condition (18) holds, with inequality (29), we have $\mathbb{E}\left(\tilde{R}_{k}\right) \leq \frac{\delta}{\|\Phi(T)\|}$, which completes the proof.

The following corollary proves that the bound given by the necessary condition will always be great than that obtained from the sufficient condition.

Corollary 4.5: For any $y \geq 0, G_{1}(y)<G_{2}(y)$, i.e. $G_{1}^{-1}(y)>G_{2}^{-1}(y)$.

Proof: Consider the equations (11) and (20-22), we have

$$
\begin{aligned}
& G_{1}(y)=2^{-h_{1}(y)}-2^{-h_{1}(y)} \frac{\ln 2}{\ln 2+\zeta_{1}(y)} \\
& G_{2}(y) \geq 2^{-h_{2}(y)}+2^{-h_{2}(y)} \ln 2 \cdot C
\end{aligned}
$$

Since $h_{1}(y)>h_{2}(y), \forall y \geq 0, C>0$ and $\zeta_{1}(y) \geq 0$, then $G_{1}(y)<G_{2}(y)$. Because $G_{1}(y)$ and $G_{2}(y)$ are both monotone increasing functions, then $G_{1}^{-1}(y)>G_{2}^{-1}(y)$ follows immediately.

The following lemma derives a sufficient condition for the selection of the controller in the low bit rate region.

Lemma 4.6: Consider the system in equation (1) with a family of control gain matrices $\left\{K_{i}\right\}_{i=1}^{N}$, suppose left tail of $R_{k}$ probability distribution satisfies the probability bound in (4) and let $A_{K}=A+B K$. If the selected controller $K \in$ $\left\{K_{i}\right\}_{i=1}^{N}$ satisfies the condition

$$
\left\|A_{K}\right\| \leq \frac{1}{T-\Delta} \ln \frac{G_{2}^{-1}\left(\frac{\delta}{\|\Phi(T)\|}\right)-\|\Phi(T)\| L_{k-1}}{\left|\hat{x}\left(a_{k-1}\right)\right|}
$$


then the necessary condition derived in lemma 4.4, i.e. $\left|x\left(\tau_{k}\right)\right| \leq G_{2}^{-1}\left(\frac{\delta}{\|\Phi(T)\|}\right), \forall k \in \mathbb{Z}$ is satisfied.

Proof: The proof is omitted since it is similar to what was used in lemma 4.3.

With the sufficient conditions in Lemma 4.4 and Lemma 4.6, we proceed to state one the paper's main theorems, which establishes the almost sure asymptotic observability for the system in equation (1).

Theorem 4.7: Given a sequence of $\left\{L_{k}\right\}_{k=0}^{\infty}$ constructed by equation (7), the encoding/decoding scheme in (5-6), and a collection of controller gain matrices $\left\{K_{i}\right\}_{i=1}^{N}$, suppose the communication channel satisfies the probability bounds (34). If there exists a sequence of switching signals $\left\{s_{k}\right\}_{k=0}^{\infty}$, in which $s_{k} \in[1, \ldots, N]$ is constant over $\left[a_{k}, a_{k+1}\right)$, such that the sufficient condition in Lemma 4.6 is satisfied under the selected controller $\left\{K_{s_{k}}\right\}_{k=0}^{\infty}$, then the system in equation (1) is almost sure asymptotically observable.

Proof: Suppose there always exists a controller $K \in\left\{K_{i}\right\}_{i=1}^{N}$ such that lemma 4.6 holds, then we have $\mathbb{E}\left(L_{k+1} \mid L_{k}\right) \leq \delta L_{k}, \forall k \in \mathbb{Z}^{+}$. This also implies [18]

$$
\begin{array}{r}
\mathbb{E}\left[L_{k}\right] \leq \delta \mathbb{E}\left[L_{k-1}\right] \leq \ldots \leq \delta^{k} L_{0} \\
\Rightarrow \mathbb{E}\left[L_{k}\right] \leq \delta^{k} L_{0}
\end{array}
$$

Since $\delta \in[0,1)$, taking the limit $k \rightarrow \infty$ leads to

$$
\underset{k \uparrow \infty}{\limsup } \mathbb{E}\left[L_{k}\right] \leq \lim _{k \uparrow \infty} \delta^{k} L_{0}=0
$$

which implies almost sure convergence $\operatorname{Pr}\left(\lim _{k \uparrow \infty} L_{k}=0\right)=$ 1. Since $L_{k}$ is the upper bound for the quantization error, the almost sure asymptotic observability immediately follows. The proof is complete.

\section{B. Almost sure asymptotic stability}

The result in Theorem 4.7 indicates that the quantization error will converge to zero with probability 1 , however, it does not say anything about the stability of the original system in equation (1). Theorem 4.9 shows that under the dwell-time condition, almost sure asymptotic observability indeed implies the almost sure asymptotic stability for the system in equation (1).

Assumption 4.8: (Dwell Time)[19], [20] Given a family of switched closed-loop system $\left\{A+B K_{i}\right\}_{i=1}^{C}$, there exists a constant fixed dwell time $\tau_{D}$ and integer $\pi \in \mathbb{Z}^{+}$, such that two consecutive switching time interval $\pi * T \geq \tau_{D}$.

Since the switching decision is always made at time instant $a_{k}$, we know the time interval between two consecutive switches must be a multiple of the sampling period $T$. One may either select large $\pi$ or large $T$ to satisfy the dwell-time assumption.

Theorem 4.9: Consider the system in (1) and suppose that there exists positive $c$ and $\lambda$ such that

$$
\left\|e^{A_{i} t}\right\| \leq c e^{-\lambda t}
$$

where $A_{i}=A+B K_{i}$ and $K_{i}$ is the $i$ th controller. If the dwell time assumption holds with $\tau_{D}>\frac{\ln c}{\lambda}$, then the system in (1) is almost sure asymptotically stable.
Proof: Without loss of generality we assume $\pi=1$ in the dwell-time assumption. Consider $t \in\left[\tau_{k}, \tau_{k+1}\right)$, we have

$$
\hat{x}\left(\tau_{k+1}^{-}\right)=e^{A_{q}(T-\Delta)} e^{A_{p} \Delta} \hat{x}\left(\tau_{k}\right)
$$

Let $E\left(\tau_{k+1}\right):=\hat{x}\left(\tau_{k+1}\right)-\hat{x}\left(\tau_{k+1}^{-}\right)$, then

$$
\hat{x}\left(\tau_{k+1}\right)=e^{A_{q}(T-\Delta)} e^{A_{p} \Delta} \hat{x}\left(\tau_{k}\right)+E\left(\tau_{k+1}\right)
$$

Taking the infinity norm on both side leads to

$$
\left|\hat{x}\left(\tau_{k+1}\right)\right| \leq\left\|e^{A_{q}(T-\Delta)}\right\|\left\|e^{A_{p} \Delta}\right\|\left|\hat{x}\left(\tau_{k}\right)\right|+\left|E\left(\tau_{k+1}\right)\right|
$$

By inserting inequality (33), we obtain

$$
\left|\hat{x}\left(\tau_{k+1}\right)\right| \leq \bar{c} e^{-\lambda T}\left|\hat{x}\left(\tau_{k}\right)\right|+\left|E\left(\tau_{k+1}\right)\right|
$$

where $\bar{c}:=c^{2}$. The term $\left|E\left(\tau_{k+1}\right)\right|$ can be further bounded by

$$
\left|E\left(\tau_{k+1}\right)\right| \leq\|\Phi(T)\| L_{k}\left(1-2^{-R_{k+1}}\right) \leq\|\Phi(T)\| L_{k}
$$

Combining inequality (36) and (37) and taking the expectation on both side gives

$$
\mathbb{E}\left(\left|\hat{x}\left(\tau_{k+1}\right)\right|\right) \leq \bar{c} e^{-\lambda T} \mathbb{E}\left(\left|\hat{x}\left(\tau_{k}\right)\right|\right)+\|\Phi(T)\| \mathbb{E}\left(L_{k}\right)
$$

Since the almost sure asymptotic observability holds, i.e. $\mathbb{E}\left(L_{k}\right) \leq \delta^{k} L_{0}$, replacing it into above inequality and propagating backwards in time, we obtain

$$
\begin{aligned}
\mathbb{E}\left(\left|\hat{x}\left(\tau_{k+1}\right)\right|\right) & \leq \bar{c}^{k+1} e^{-\lambda T(k+1)} \mathbb{E}\left(\left|\hat{x}\left(\tau_{0}\right)\right|\right) \\
& +L_{0}\|\Phi(T)\| \delta^{k} \sum_{i=0}^{k}\left(\frac{\bar{c} e^{-\lambda T}}{\delta}\right)^{i} \\
& \leq e^{(k+1)(\ln \bar{c}-\lambda T)} \mathbb{E}\left(\left|\hat{x}\left(\tau_{0}\right)\right|\right) \\
& +\frac{\delta^{k+1}-e^{(k+1)(\ln \bar{c}-\lambda T)}}{\delta-e^{(\ln \bar{c}-\lambda T)}} L_{0}\|\Phi(T)\|
\end{aligned}
$$

Here, if the switching time interval $T>\frac{\ln \bar{c}}{\lambda}$, then

$$
\lim _{k \rightarrow \infty} \mathbb{E}\left(\left|\hat{x}\left(\tau_{k}\right)\right|\right) \rightarrow 0
$$

With $\lim _{k \rightarrow \infty} \mathbb{E}\left(L_{k}\right) \rightarrow 0$, the almost sure asymptotic stability for the original system is achieved, which completes the proof.

\section{Simulation}

In this section, a vehicle to vehicle tracking example is used to demonstrate lemma 4.4 and lemma 4.6 which provide the sufficient conditions for almost sure asymptotic stability. The simulation compares two control strategies, one is using lemma 4.6 to switch the controller in response to the changes in communication channel state, while the other one is applying a non-switching control strategy without using the channel information. 


\section{A. vehicle tracking model}

Consider the following vehicle model,

$$
\begin{aligned}
\dot{y}_{i} & =v_{i} \\
\dot{v}_{i} & =u_{i}, i=1,2
\end{aligned}
$$

where $y_{i}$ and $v_{i}$ represent the longitudinal position and velocity for each vehicle. $u_{i}$ is the control policy used by each vehicle to manage their accelerations. The control objective is to maintain a specified separation distance between the leader and follower, and achieve velocity tracking for the follower. To make the tracking problem nontrivial, we consider the case that the leader changes its control profile over time. The control input $u_{1}$ to the leader is a piecewise constant signal, which models a vehicle traveling on road sections with different speed limits. We assume the leader's control profile changes slowly enough to be perfectly known by the follower. We can therefore develop the following model for the tracking error,

$$
\begin{array}{r}
\dot{\eta}_{1}=\eta_{2} \\
\dot{\eta}_{2}=\bar{u}
\end{array}
$$

with $\eta_{1}=y_{1}-y_{2}-L_{s}, \eta_{2}=v_{1}-v_{2}$ and $\bar{u}=u_{1}-u_{2} . L_{s}$ denotes the safe distance margin for the two vehicles. Since $u_{1}$ is known to the follower, it can be treated as a known disturbance. As we can see in the tracking model, in order to achieve the control objective, The leader must transmit its longitudinal position $\left(y_{1}\right)$ and velocity $\left(v_{1}\right)$ to the follower in order to meet the control objectives. This transmission is done over a wireless fading channel. which satisfies the probabilistic bounds (3-4).

\section{B. Simulation setup}

A two-state Markov chain model is used to simulate the communication channel between the two vehicles. One state represents the bad channel condition, another one represents the good channel condition. The bad channel condition is characterized in terms of those channel states satisfying $R_{k} \leq 1.8 e^{-10^{-4}|\eta|^{2}}$, while the good channel condition is $R_{k} \geq$ $2.4 e^{-10^{-4}|\eta|^{2}}$. The probability associated with the bad state is $p_{11}(\mid \eta \|)=e^{-1.8 e^{-0.0251|\eta|^{2}}}$, while the probability for the good state is $p_{22}(|\eta|)=e^{-2.4 e^{0.009|\eta|^{2}}}$. The corresponding transition probabilities are $1-p_{11}(|\eta|)$ and $1-p_{22}(|\eta|)$. Under these assumptions on the Markov chain, one can then evaluate the bounds in equations (3-4) as

$$
\begin{aligned}
& h_{1}(|\eta|)=2.4 e^{-10^{-4} \|\left.\eta\right|^{2}}, h_{2}(|\eta|)=1.8 e^{-10^{-4} \|\left.\eta\right|^{2}} ; \\
& \zeta_{1}(|\eta|)=e^{0.001|\eta|^{2}}, \zeta_{2}(|\eta|)=e^{-0.025|\eta|^{2}} .
\end{aligned}
$$

The other simulation parameters are set as follows

$$
\begin{array}{r}
T=0.1, \Delta=0.02, \bar{R}=2, \delta=0.8 ; \\
K_{1}=[-1-1] ; K_{2}=[-36-12]
\end{array}
$$

By Lemma 4.4, the state set assuring specific performance is determined as $|\eta| \leq G_{2}^{-1}\left(\frac{\delta}{\|\Phi(T)\|}\right)=20.86$. The initial state $(20,10)$ is selected to satisfy the sufficient condition. Two controllers $K_{1}=[-1-1] ; K_{2}=[-36-12]$ are selected to ensure different performance levels. $K_{1}$ is the controller with small gain, which leads to slow response time but is less sensitive to the uncertainty. $K_{2}$ is the controller with high gain, which leads to fast response times but is more sensitive to uncertainty.

A Monte Carlo method is used to verify the almost sure asymptotic stability of the system. The simulation is run 1000 times over the time interval from 0 to 10 seconds. Figure 4 shows the maximum and minimum values of each state $\eta_{i}, i=1,2$ as a function of time over all 1000 runs. The top plot in the figure is the trajectory for state $\eta_{1}$, with maximum value marked as blue solid line, and minimum value marked as red dash line. The black line represents the sufficient bound on the state derived from lemma 4.4. The bottom plot is for the state $\eta_{2}$. We can see from the plots that the maximum and minimum values of the system state converge to zero almost surely after about 3.5 seconds. These results are consistent with our statement in lemma 4.4 that establishes the almost sure asymptotic stability of the switched system.

We also studied the benefits of switching controllers in response to the changes of channel state. Two control strategies were compared in this simulation. One is the non-switching policy, which applies the conservative controller $K_{1}$ all the time without considering the changes of channel state. The other one is the switching control policy that switches the controller in response to the changes of channel state to compensate the deep fading and achieve a fast convergent rate.

In figure 5, the top plot shows the channel state $\left(R_{k}\right)$ profile along the time line. The bottom plot is the switching policy that reacts to the changes of channel state. We can see from the top plot that there is a string of zero bits over the time interval $[0,2]$ which represents a deep fading scenario. At that time interval, the switching policy selects the conservative controller (index 1 ) to make a safe control decision. Shortly after 2 seconds, the channel state switches to the high bit rate, which makes the system switch to the high gain controller (index 2) to achieve better performance. The channel exhibits another long string of zero bits which is shown between time interval $[2,4]$. The system detects this deep fade and switches back to small gain controller. After about 3.5 seconds, the high gain controller is always switched on because the channel condition remains good.

Figure 6 shows the system trajectories for both switching policy and non-switching policy. The top plot in the figure shows the comparison for state $\eta_{1}$, while the bottom one is for $\eta_{2}$. The blue solid line represents the trajectory generated by switching policy while the red dashed one is by nonswitching policy. As shown in the figure, the system performance is comparatively the same for both control policies before 3.5 seconds, since both of them are using low control gain. After 3.5 seconds, the switching policy selects the high gain controller. The state converges to the equilibrium at around 4 seconds for the switching strategy, while it takes more than 8 seconds for the non-switching one to converge. 

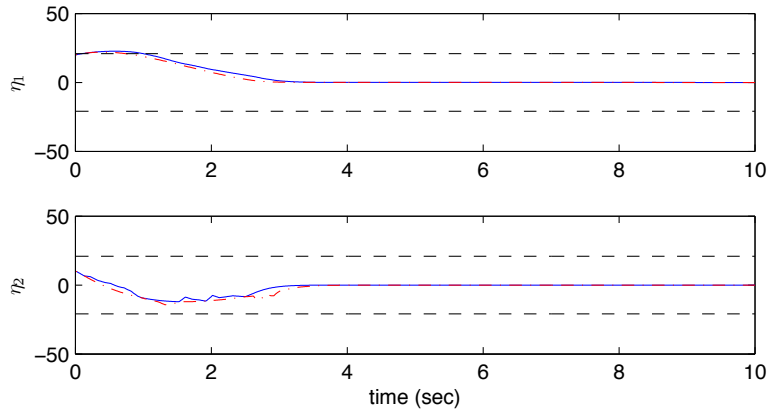

Fig. 4. Almost sure asymptotic stability with switching strategy
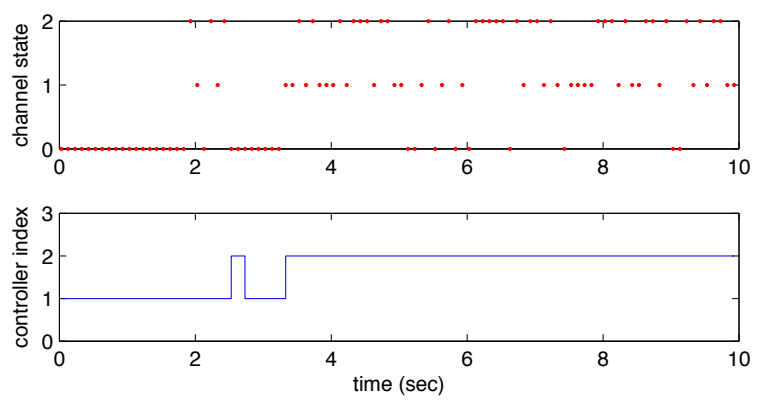

Fig. 5. The switching policy in response to the changes of channel state

Although non-switching control policy can preserve safety in the deep fading scenario, it sacrifices system performance when the channel condition is good.

\section{CONCLUSION}

This paper studies the almost sure asymptotic stability of the control system in the presence of wireless fading channel whose outages are a function of the system state. Necessary and sufficient conditions are established for a switching controller that assures almost sure asymptotic stability. Preliminary simulations results support the analysis.

\section{ACKNOWLEDGMENT}

The authors acknowledge the partial financial support of the National Science Foundation (NSF-CNS-1239222).

\section{REFERENCES}

[1] P. Varaiya, "Smart cars on smart roads: problems of control," Automatic Control, IEEE Transactions on, vol. 38, no. 2, pp. 195-207, 1993.

[2] C. Rieger, D. Gertman, and M. McQueen, "Resilient control systems: next generation design research," in Human System Interactions, 2009. HSI'09. 2nd Conference on. IEEE, 2009, pp. 632-636.

[3] D. Tse and P. Viswanath, Fundamentals of wireless communication. Cambridge university press, 2005.

[4] H. Wang and N. Moayeri, "Finite-state markov channel - a useful model for radio communication channels," IEEE Transactions on Vehicular Technology, vol. 44, no. 1, pp. 163-171, 1995.

[5] A. S. Akki and F. Haber, "A statistical model of mobile-to-mobile land communication channel," IEEE Transactions on Vehicular Technology, vol. 35 , no. 1 , pp. $2-7,1986$.

[6] C. S. Patel, G. L. Stuber, and T. G. Pratt, "Simulation of rayleighfaded mobile-to-mobile communication channels," IEEE Transactions on Communications, vol. 53, no. 11, pp. 1876-1884, 2005.
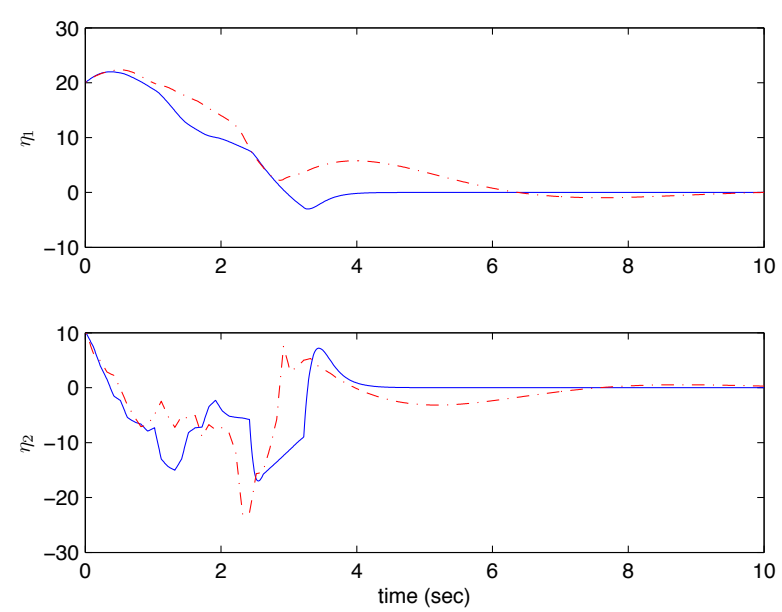

Fig. 6. The state trajectory with switching strategy and non-switching strategy

[7] L. Cheng, B. Henty, F. Bai, and D. D. Stancil, "Doppler spread and coherence time of rural and highway vehicle-to-vehicle channels at 5.9 ghz," in Proc. IEEE Global Telecommunications Conf. IEEE GLOBECOM 2008, 2008, pp. 1-6.

[8] L. Cheng, B. E. Henty, D. D. Stancil, F. Bai, and P. Mudalige, "Mobile vehicle-to-vehicle narrow-band channel measurement and characterization of the $5.9 \mathrm{ghz}$ dedicated short range communication (dsrc) frequency band," IEEE Journal on Selected Areas in Communications, vol. 25, no. 8, pp. 1501-1516, 2007.

[9] W. S. Wong and R. W. Brockett, "Systems with finite communication bandwidth constraints. ii. stabilization with limited information feedback," IEEE Transactions on Automatic Control, vol. 44, no. 5, pp. 1049-1053, 1999.

[10] R. W. Brockett and D. Liberzon, "Quantized feedback stabilization of linear systems," IEEE Transactions on Automatic Control, vol. 45, no. 7, pp. 1279-1289, 2000.

[11] N. Elia and S. K. Mitter, "Stabilization of linear systems with limited information," IEEE Transactions on Automatic Control, vol. 46, no. 9, pp. 1384-1400, 2001.

[12] S. Tatikonda and S. Mitter, "Control under communication constraints," IEEE Transactions on Automatic Control, vol. 49, no. 7, pp. 1056-1068, 2004.

[13] G. N. Nair, R. J. Evans, I. M. Y. Mareels, and W. Moran, "Topological feedback entropy and nonlinear stabilization," IEEE Transactions on Automatic Control, vol. 49, no. 9, pp. 1585-1597, 2004.

[14] N. C. Martins, M. A. Dahleh, and N. Elia, "Feedback stabilization of uncertain systems in the presence of a direct link," IEEE Transactions on Automatic Control, vol. 51, no. 3, pp. 438-447, 2006.

[15] P. Minero, M. Franceschetti, S. Dey, and G. N. Nair, "Data rate theorem for stabilization over time-varying feedback channels," IEEE Transactions on Automatic Control, vol. 54, no. 2, pp. 243-255, 2009.

[16] O. Yaron and M. Sidi, "Performance and stability of communication networks via robust exponential bounds," IEEE/ACM Transactions on Networking, vol. 1, no. 3, pp. 372-385, 1993.

[17] D. Starobinski and M. Sidi, "Stochastically bounded burstiness for communication networks," Information Theory, IEEE Transactions on, vol. 46, no. 1, pp. 206-212, 2000.

[18] J. Doob, Stochastic Processes. John Wiley \& Sons, 1953.

[19] J. P. Hespanha and A. S. Morse, "Stability of switched systems with average dwell-time," in Proc. 38th IEEE Conf. Decision and Control, vol. 3, 1999, pp. 2655-2660.

[20] D. Liberzon, "Stabilizing a switched linear system by sampled-data quantized feedback," in Proc. 50th IEEE Conf. Decision and Control and European Control Conf. (CDC-ECC), 2011, pp. 8321-8326. 